\title{
Adsorption of Benzene on Rice Husk-based Activated Carbons with Low Silicon Content
}

\author{
Fuyong Wang ${ }^{1}$, Qiang Yư ${ }^{1}$, Wenhua Ji ${ }^{1}$, Lixia Liu ${ }^{1}$, Li Yang ${ }^{1}$ and Dongli Fanª, \\ ${ }^{1}$ Suzhou institute of supervision \&inspection on product quality,suzhou,jiangsu 215000, China \\ ${ }^{2}$ Nantong university, College of chemistry and chemical engineering, Nantong, Jiangsu \\ 226000, China \\ afdlsky@163.com
}

Keywords: Rice husk; Activate; Activated carbon; Adsorption; Heating

\begin{abstract}
The activated carbon with high specific surface area and high content of mesopore is prepared, with rice husk as raw material, $\mathrm{NaOH}$ as activing agent.The effects of such factors as the ratio of phosphate to rice husks, the activating temperature and time, heating rate and different activation process upon the adsorption properties of active carbon for benzene were investigated. The results indicate that the activated carbon obtained at phosphoric acid/carbon ratio of 1:2.5; heating rate of $300^{\circ} \mathrm{C} / \mathrm{h}$, activation temperature of $500^{\circ} \mathrm{C}$, the activating time of $3 \mathrm{~h}$ exhibits the best adsorption properties of active carbon for benzene which reached $130 \%$.
\end{abstract}

\section{Introduction}

Activated carbon (AC) products are produced from organic materials that are rich in carbon. In China and many parts of the world, a large amount of rice husk could be obtained as an agricultural by-product during rice production.Farmers on-site burning caused severe environmental pollution and the waste of resources. Therefore, a large number of researchers have been directed toward the utilization of waste materials.Nowadays, waste materials or pozzolans from industrial and agricultural by-products such as fly ash and rice husk ash are receiving more attention[1-3].

Rice husk ash is high in silica content in the form of noncrystalline or amorphous material silica.By burning the rice husk under the controlled temperature and atmosphere, a highly reactive rice husk ash is obtained.The activated carbon which prepared by chemical activation have well-developed porosity and large specific surface area.In this experiment,active carbon was prepared from rice husk, which was treated with phosphoric acid[4-5]. The effects of such factors as the ratio of phosphate to rice husks, the activating temperature and time, heating rate and different activation process upon the adsorption properties of active carbon for benzene were investigated.

\section{Experimental}

Materials and Reagents. Rice husk was provided by a rice mill in Nantong, China, in the autumn of 2014.Previous to carbonization process, rice husk was washed thoroughly with distilled water to remove the impurities. And then the rice husk was dried in an oven at $100^{\circ} \mathrm{C}$ for $24 \mathrm{~h}$ to a constant weight. The material used as the precursor of the activated carbon was stored in dry and ventilated place before use.All chemicals and solvents used in this study were analytical grade and used without further purification.Phosphoric acid, $\mathrm{NaOH}$,benzene and ammonium hydroxide(allanalytical grade)were provided by Tianjin Kermel Co.Distilled water was applied for all synthesis and treatment processes. 
Production of Activated Carbon. Alkali hydroxides are generally used as activating agents for the production of ACs because of the valuable properties of materials produced by this activator. In particular, porosity development by alkali hydroxide activation is associated with a well-established gasification mechanism. Thus, in this study, the activated carbons was mixed with a certain amount of sodium hydroxide solution in the three necked flask and the inorganic components ( $\mathrm{SiO} 2)$ were removed from the rice husk by washing with $\mathrm{NaOH}$. The impregnation ratio was 1:3 and to evaluate the influence of activating agent during the impregnation,impregnation in both materials, raw $\mathrm{RH}$ and carbonized RH, was also conducted.The varnished Rice husk was mixed with $85 \mathrm{wt} \%$ phosphoric acid, the concentration ratios of phosphoric acid to rice husk were from 1:1 to 3:1. The activation reactions were carried out at the high temperature ranging from $400^{\circ} \mathrm{C}$ to $700^{\circ} \mathrm{C}$ with the heating rate $600^{\circ} \mathrm{C} / \mathrm{h} 、 300^{\circ} \mathrm{C} / \mathrm{h} 、 150^{\circ} \mathrm{C} / \mathrm{h}$ and maintained for $2-4 \mathrm{~h}$. The product was thoroughly washed with hot distilled water to remove the excess phosphorus compounds until the wash water attained $\mathrm{pH}$ 7. The washed activated carbon samples were dried at $110{ }^{\circ} \mathrm{C}$ for $12 \mathrm{~h}$, ready for adsorption test.

Dissolution Rate of $\mathrm{SiO}_{2}$. The titration method is used to measure the content of $\mathrm{Na} 2 \mathrm{SiO} 3$ for the evaluate of the dissolution rate of $\mathrm{SiO} 2$, because $\mathrm{SiO} 2$ has already dissolved in $\mathrm{NaOH}$ solution. The mother liquor of the boiling rice husk were taken $(10 \mathrm{~mL})$ respectively into conical flask $\mathrm{A}, \mathrm{B}, \mathrm{C}$, and the indicator was methyl orange. Unreacted $\mathrm{NaOH}$ was titrated by $\mathrm{HCl}$ solution $(0.5 \mathrm{~mol} / \mathrm{L})$, and the initial reading was $\mathrm{V} 1$. When the color of the solution turned into reddish, the titration endpoint was reached and the burette reading was V2.

About $15 \mathrm{ml} 0.5 \mathrm{~mol} / \mathrm{L} \mathrm{NaF}$ solution (excessive)was dropwise added into the conical flask, the color of the solution turned from reddish to yellow, and then $0.5 \mathrm{~mol} / \mathrm{L} \mathrm{HCl}$ was used to titrate $\mathrm{NaOH}$ what was produced by reaction. The initial reading was V3 and when the color of the solution turned into reddish, the titration endpoint was reached, the burette reading was V4. Meanwhile,the beaker was thoroughly washed with distilled water to remove the impurities until the wash water attained $\mathrm{pH}$, and then dried to a constant weight $(\mathrm{m} 2)$ by oven. Dissolution rate of $\mathrm{SiO}_{2}=\left(V_{4}-V_{3}\right) \times c_{H C l} \div 4 \times M_{\mathrm{SiO}_{2}}$

$$
\text { Where } c_{\mathrm{HCl}}=0.5 \mathrm{~mol} / \mathrm{L}, M_{\mathrm{SiO}_{2}}=60 \mathrm{~g} / \mathrm{mol}
$$

Adoption. Equilibrium adoption studies were conducted in a set of pre-dried empty weighing bottles containing adsorbent. These bottles were dried in a vacuum oven for $2 \mathrm{~h}$ and put in benzene steam cylinder to adsorb benzene steam until the equilibrium was reached.

Benzene adsorption value $=(\mathrm{m} 3-\mathrm{m} 2) /(\mathrm{m} 2-\mathrm{m} 1)$

Where $\mathrm{m} 1$ is the weight of empty weighing bottle after drying, $\mathrm{m} 2$ is the whole weight of bottle and activated carbon before adsorption, $\mathrm{m} 3$ is the whole weight of bottle and activated carbon after saturated adsorption.

\section{Results and Discussion}

The Dissolution Rate of Silica. The effect of the dissolution rate of $\mathrm{SiO} 2$ on the boiling time was shown in Fig. 1 which calculated according to the Eq. 1.

As can be seen from Fig. 1, the longer the rice husk boiled in $\mathrm{NaOH}$ solution, the higher the rate of dissolution of $\mathrm{SiO} 2$. The ratio tended to balance after soaking for $5 \mathrm{~h}$. It was found that the dissolution rate of $\mathrm{SiO} 2$ was only $3 \%$ if just soak the rice husk in $\mathrm{NaOH}$ solution at room temperature for $24 \mathrm{~h}$ without heating, and the effect of boiling for $3 \mathrm{~h}$ was almost the same.so, the 
effect was better when the rice husk boiled in $\mathrm{NaOH}$ solution.isoak visible boiled rice husk with $\mathrm{NaOH}$ better. Therefore the optimal boiling time was $5 \mathrm{~h}$.

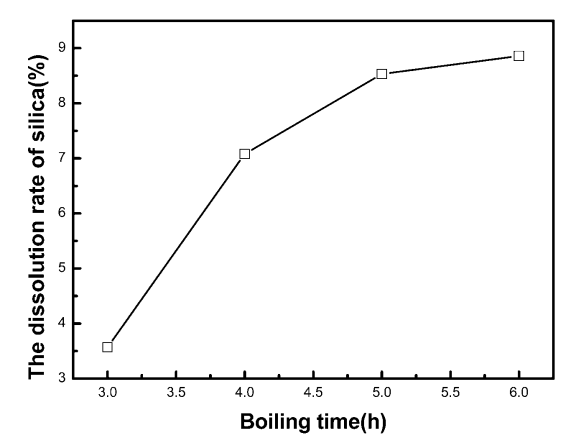

Figure 1. Effect of the dissolution rate of $\mathrm{SiO} 2$ on the boiling time

Effect of Dissolution Rate. The effects of the dissolution rate of $\mathrm{SiO} 2$ of rice husk on the absorbing properties of benzene of rice husk-based activated carbon were studied under the following condition: the phosphate concentration ratio $1: 2$, heating rate $300{ }^{\circ} \mathrm{C} / \mathrm{h}$, the activation temperature $500{ }^{\circ} \mathrm{C}$ and the activation time $3 \mathrm{~h}$. The determination of absorbing properties was proceeded under the normal temperature and pressure and the results were shown in Fig. 2.

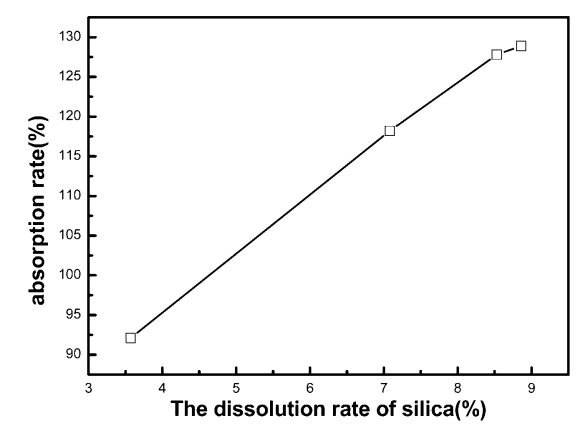

Figure 2.Effect of dissolution rate

As can be seen from Fig. 2, the more the dissolution rate of $\mathrm{SiO} 2$, the higher the rate of activated carbon adsorption. The main reason for these phenomena could be explained as follows:The fewer content of $\mathrm{SiO} 2$ in rice husk, the less inorganic impurities in activated carbon, and the adsorption rate of activated carbon increased. In addition, $\mathrm{SiO} 2$ and other inorganics in rice husk would not become small molecules and evaporate during the process of carbonization. They will always exist in the activated carbon and $\mathrm{SiO} 2$ may block some formed pores and thereby reducing the surface area of activated carbon and decreasing the adsorption rate of activated carbon.

Effect of the Phosphate Concentration Ratio. The effects of the phosphate concentration ratio on the absorbing properties of benzene of rice husk-based activated carbon were studied under the following condition: heating rate $300{ }^{\circ} \mathrm{C} / \mathrm{h}$, the activation temperature $500{ }^{\circ} \mathrm{C}$ and the activation time 3 h. The results were shown in Fig. 3. 


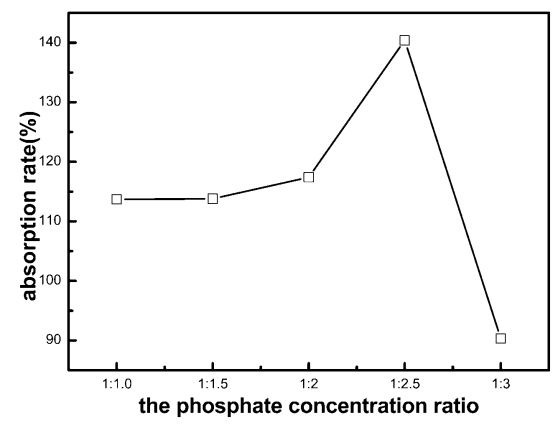

Figure 3. Effect of the phosphate concentration ratio

As can be seen from Fig. 3, the adsorption ratio of activated carbon increased with the increase of phosphate concentration ratio and get the maxmium at the concentration ratio of 1:2.5.This is because the catalysis of the phosphoric acid which prompting the decomposition of hydroxyl groups of rice husk and the dehydration reaction. Then the formation of carbon volatiles in the rice husk inhibited and more carbon was reserved in the solid phase, thus the adsorption rate of activated carbon was enhanced. But when the concentration ratio of phosphoric acid is too large (>1:5), the adsorption rate of activated carbon will reduce greatly. Two possible reasons are as follows: first, the surface of rice husk will be surrounded by dense phosphate molecules when the phosphoric acid concentration was too large.Because of the flame retardant effect of phosphate, the decomposition of organics in rice husk inhibited, and adsorption rate of the activated carbon decreased. Second, the tar which produced in the process of thermal decomposition of rice husk will generate pores clogging, and thus to lessen the number of effective air gap and finally reduce the specific surface area of activated carbon and the adsorption capacity of activated carbon. So the optimum phosphate concentration ratio was $1: 2.5$.

Effect of Heating Rate. The effects of the heating rate on the absorbing properties of benzene of rice husk-based activated carbon were studied under the following condition: the phosphate concentration ratio 1: 2 , the activation temperature $500{ }^{\circ} \mathrm{C}$ and the activation time $3 \mathrm{~h}$. The results were shown in Fig. 4.

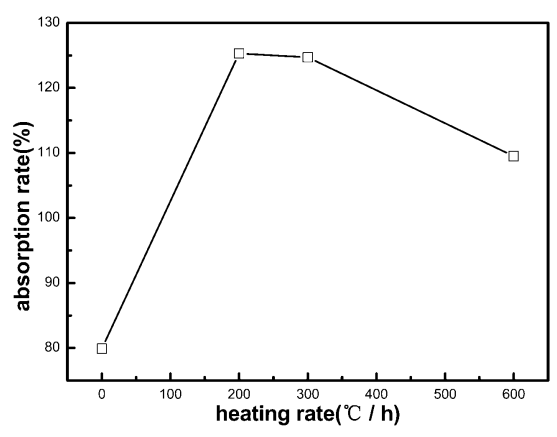

Figure 4.Effect of heating rate

As the results shown in Fig. 4, the adsorption rate of activated carbon increased gradually when the heating rate of activation temperature was slow. The main reason was just as follows: The carbonization was completely and ran smoothly because of the slowly heating rate. The volatiles in the rice husk and the gas which generated in the reaction escape slow, and this was advantageous to the formation of more pore in the rice husk. In addition, the slowly heating rate is also means the extending of the activation time which makes the rice husk ablated constantly and the pores kept 
getting bigger. So the adsorption capacity of activated carbon increased[6]. It can also be seen from the Fig. 4, with the continuous reduction of heating rate, the upward trend of adsorption rate slowed down. So the feasible heating rate was $300{ }^{\circ} \mathrm{C} / \mathrm{h}$.

Effect of Activation Temperature. The effects of the activation temperature on the absorbing properties of benzene of rice husk-based activated carbon were studied under the following condition: the phosphate concentration ratio $1: 2$, heating rate $300{ }^{\circ} \mathrm{C} / \mathrm{h}$, the activation time $3 \mathrm{~h}$. The results were shown in Fig. 5.

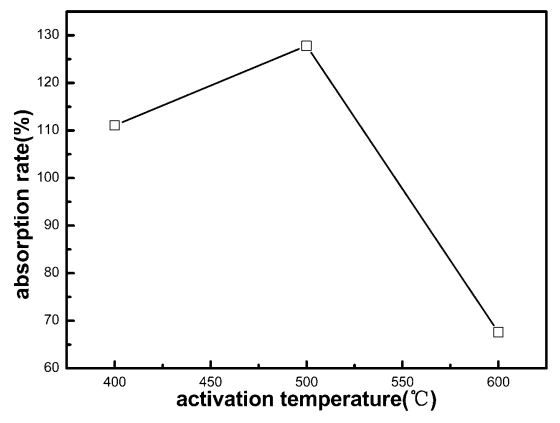

Figure 5.Effect of activation temperature

As the results shown in Fig. 5, when the temperature was less than $500{ }^{\circ} \mathrm{C}$, with the increase of temperature, the reaction in the process of carbonization was more completely, the pore volumn in activated carbon increased and the specific surface area was larger. so the adsorption rate of activated carbon will be gradually increased as the temperature increases. While the adsorption rate of activated carbon decreased when the temperature was higher than $500{ }^{\circ} \mathrm{C}$ ( the rice husk has been basically incinerated when the temperature is $700{ }^{\circ} \mathrm{C}$ ). This was because the formed pores continued to react with the activating agent and the diameter of the hole increased gradually which reducing the specific surface area of the activated carbon and lowering the adsorption rate of the activated carbon. In addition, if the temperature was too high, the rice husk was easily burned in the process of carbonization and the "ablation" rate is relatively high, so the adsorption rate of activated carbon will increase first and then decreased as the temperature rises[7,8].

Effect of Activation Time. The effects of the activation time on the absorbing properties of benzene of rice husk-based activated carbon were studied under the following condition: the phosphate concentration ratio $1: 2$, heating rate $300{ }^{\circ} \mathrm{C} / \mathrm{h}$, the activation temperature $500{ }^{\circ} \mathrm{C}$. The results were shown in Fig. 6.

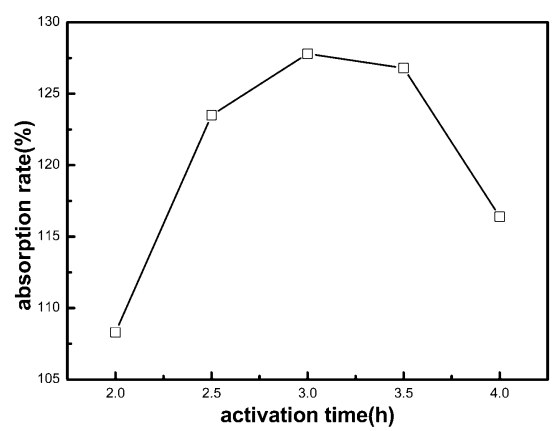

Figure 6. Effect of activation time 
As can be seen from Fig. 6, with the increase of the activation time, the adsorption rate of activated carbon is gradually increased. It mainly because the organic matter in rice husk has been broken down and the fine pore structure has been formed under the effect of phosphoric acid and high temperatures. The adsorption rate of activated carbon decreased slowly when the activation time is more than $3 \mathrm{~h}$, while decreased rapidly if more than $3.5 \mathrm{~h}$. This shows that when the activation time is more than $3 \mathrm{~h}$, the ablation rate of rice husk increased gradually in the process of carbonization, and the diameter of the pores which formed in the process of activation increased gradually with the increase of the activation time. Thereby the surface area of activated carbon reduced and the adsorption ratio of activated carbon began to decrease[9,10]. So the feasible activation time was $3 h$.

\section{Conclusion}

The results obtained showed that rice husk-based activated carbon with low silicon content can be readily used for the adsorption of benzene.The adsorption rate of activated carbon increased with the increase of the dissolution rate of $\mathrm{SiO} 2$ from rice husk. The adsorption of activated carbon can be controlled by modification of the impregnation ratio of phosphoric acid, activation temperature, activation time and heating rate. The optimum activation temperature is $500^{\circ} \mathrm{C}$, activation time is $3 \mathrm{~h}$, the impregnation ratio is $1: 2.5$, heating rate is $300^{\circ} \mathrm{C} / \mathrm{h}$.

\section{References}

[1] Sumrerng Rukzon, Prinya Chindaprasirt, and Rattana Mahachai,Effect of grinding on chemical and physical properties of rice husk ash[J],International Journal of Minerals, Metallurgy and Materials, 2009,16(2):242-247

[2] Dimitrios Kalderis, Dimitrios Koutoulakis, Panagiota Paraskeva,et al,Adsorption of polluting substances on activated carbons prepared from rice husk and sugarcane bagasse[J],Chemical Engineering Journal ,2008,14:42-50

[3] Kairan Zhu, Hao Fu, Jinghui Zhang,et al,Studies on removal of NH4+-N from aqueous solution by using the activated carbons derived from rice husk[J],biomass and bioenergy,2012,43:18-25

[4] Long Lin,Shang-Ru Zhai,Zuo-Yi Xiao et al,Dye adsorption of mesoporous activated carbons produced from $\mathrm{NaOH}$-pretreated rice husks[J],Bioresource Technology,2013,136:437-443

[5] Wang Qibao,Zhang Xianglan,Xu Deping,et al.Effect of Pre-oxidation on the Properties of Crushed Bituminous Coal and Activated Carbon Prepared Therefrom[J].Journal of China of University of Mining \& Technology.2007,17(4):494-497.

[6] Peng Cheng,Zhang Zhen,Cheng Xuan,et al. Comparative studies of adsorbed CO and methanol electrooxidation oncarbon supported $\mathrm{Pt}$ and $\mathrm{PtRu}$ catalysts in acid solution[J]. RARE METALS.2006,25(3):274-280.

[7] Huijie Zhu,Yuanhong Wang,Guozhen Wang et al. Removal of Fluorine from Water by the Aluminum-Modified Activated Carbon[J]. Conference on Environmental Pollution and Public Health,2010:1127-1130.

[8] SHAN Xiaomei,ZHU Shuquan,ZHANG Wenhui.Effect of surface modification of activated carbon on its adsorption capacity for $\mathrm{NH} 3[\mathrm{~J}]$.China University Ming\&Technol.2008,18:261-265 
[9] S Somasundaram, K Sekar, VK Gupta, S Ganesan.Synthesis and characterization of mesoporous activated carbon from rice husk for adsorption of glycine from alcohol-aqueous mixture[J].Journal of Molecular Liquids, 2013, 177(8):416-425

[10]SM Yakout.Adsorption kinetics modeling of benzene from aqueous solution onto rice husk activated carbon prepared by $\mathrm{H} 3 \mathrm{PO} 4$ activation[J].Journal- Indian Chemical Society, 2014, 91(10):1915-1924 